\title{
Common Carotid Artery Laceration and Innominate Artery Pseudo-Aneurysm Following a Percutaneous Dilatational Tracheostomy Attempt
}

\author{
Parag A Brahmbhatt MD, Fagun D Modi MD, Thomas M Roy MD, and Ryland P Byrd Jr MD
}

\begin{abstract}
Percutaneous dilatational tracheostomy (PDT) has become an appropriate alternative to conventional surgical tracheostomy. It is now performed worldwide by a diverse array of physician specialists. Although adverse events are relatively uncommon, serious complications can arise from this bedside procedure. We report a patient who suffered life-threatening hemorrhage from a common carotid artery laceration and pseudo-aneurysm formation in the innominate artery following an elective PDT procedure. Key words: percutaneous dilatational tracheostomy; common carotid artery laceration; innominate artery pseudo-aneurysm. [Respir Care 2014;59(10):e153-e155. (C) 2014 Daedalus Enterprises]
\end{abstract}

\section{Introduction}

There are several documented complications associated with percutaneous dilatational tracheostomy (PDT). These complications are typically categorized as early or late depending on whether they manifest within the first week following the procedure or later. The previously described early complications include localized bleeding, mucus plugging, tracheitis, and accidental decannulation. ${ }^{1}$ Localized bleeding is often associated with vascular anomalies. To our knowledge, carotid artery laceration has not been described as a complication of PDT. In addition, innominate artery pseudo-aneurysm formation has not been previously reported as a complication of PDT. We report the occurrence of both of these vascular injuries in a patient as early complications of PDT.

\footnotetext{
Dr Brahmbhatt is affiliated with the Department of Internal Medicine, and Dr Modi is affiliated with the Division of Pulmonary Diseases and Critical Care Medicine, East Tennessee State University, Johnson City, Tennessee. Drs Roy and Byrd are affiliated with the Division of Pulmonary Diseases and Critical Care Medicine, James H. Quillen Veterans Affairs Medical Center, Mountain Home, Tennessee.
}

The authors have disclosed no conflicts of interest.

Correspondence: Ryland P Byrd Jr MD, Division of Pulmonary Diseases and Critical Care Medicine, Veterans Affairs Medical Center, 111-B, PO Box 4000, Mountain Home, TN 37684-4000. E-mail: ryland.byrd@ med.va.gov.

DOI: $10.4187 /$ respcare. 02715

\section{Case Report}

A 57-y-old male patient had undergone an amputation below the left knee due to osteomyelitis of the foot. His past history was significant for coronary artery disease, ischemic cardiomyopathy, anemia, and diabetes mellitus with neuropathy. His body mass index was $19 \mathrm{~kg} / \mathrm{m}^{2}$. Central venous access had not been placed or attempted during hospitalization for this procedure. In addition, the patient could not recall ever having a central venous catheter placed.

He was recovering in a nursing home and receiving physical and occupational rehabilitation and nutritional support. His nursing home stay was complicated by methicillin-resistant Staphylococcus aureus pneumonia and a multidrug-resistant urinary tract infection. He had received antimicrobial therapy for these infectious events by peripheral intravenous access. He became hypotensive, necessitating transfer to the ICU for septic shock. The patient subsequently developed hypoxic respiratory failure requiring intubation and prolonged mechanical ventilator support. For this reason, PDT was considered appropriate. No deep venous access had been attempted or placed during this hospitalization.

PDT was initiated with a small midline transverse incision between the second and third tracheal rings by experienced attending surgeons. Neither preoperative nor intraoperative ultrasound of his neck was performed. The introducer needle was inserted into the trachea on the first attempt under direct visualization using a flexible fiberoptic bronchoscope. At this point, the bronchoscope was re- 


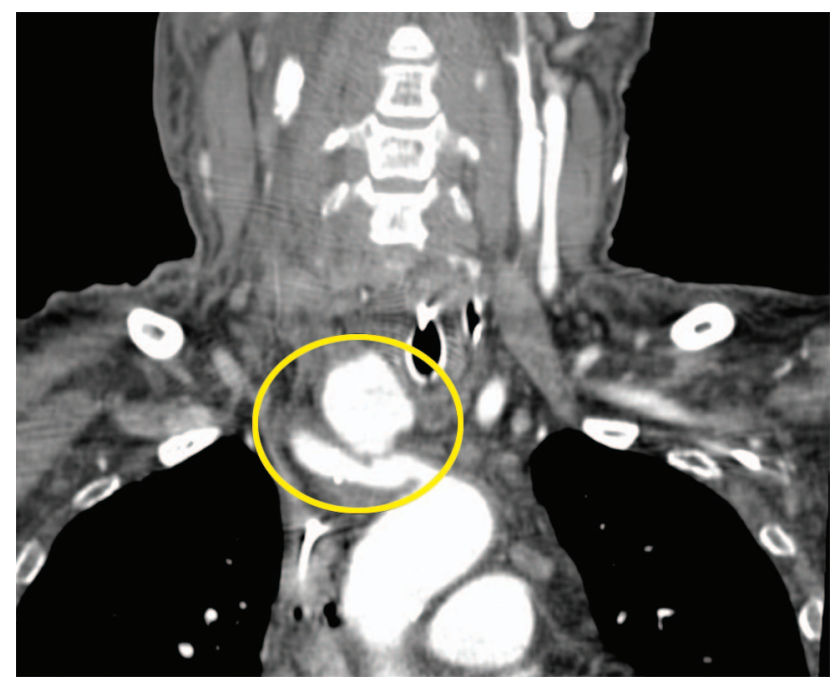

Fig. 1. Coronal computed tomogram image demonstrating a pseudo-aneurysm of the right innominate artery (circle).

moved. Two successive dilators were then passed into the trachea. Up to this point, the PDT had proceeded without difficulty. The patient began to bleed profusely as a No. 8 tracheostomy tube was being placed over the second dilator. The PDT was immediately terminated. The patient's neck was surgically explored, and a lacerated right common carotid artery was identified as the site of the bleeding. Vertical mattress sutures controlled the hemorrhaging. The patient received transfusions of 6 units of packed red blood cells. There was no further evidence of bleeding after the surgical repair of the common carotid artery laceration. After stabilizing the patient in the operating room, the patient was transferred to the ICU for continuation of care. His airway was not re-examined via endoscope. Computed tomography angiography of the great vessels of his neck was performed on the day of the PDT attempt. No bleeding site was identified. However, a right innominate artery pseudo-aneurysm was discovered (Figs. 1 and 2). No anomalous arteries were identified. No pulsatile mass, palpable thrill, or evidence of superior vena cava syndrome was present on physical examination. Two $d$ later, the patient underwent successful repair of the pseudo-aneurysm, as well as a conventional tracheostomy. Unfortunately, the patient died after a prolonged hospitalization from multi-organ failure.

\section{Discussion}

Minor bleeding during PDT has been reported to occur in fewer than $20 \%$ of cases. Bleeding is most common in the early postoperative period and may be more prevalent in PDT compared with conventional tracheostomy. Major bleeding has occurred in fewer than 5\% of cases of PDT but is typically venous. ${ }^{2}$ Catastrophic hemorrhage, as experienced in our patient, is rare. Most of the cases of

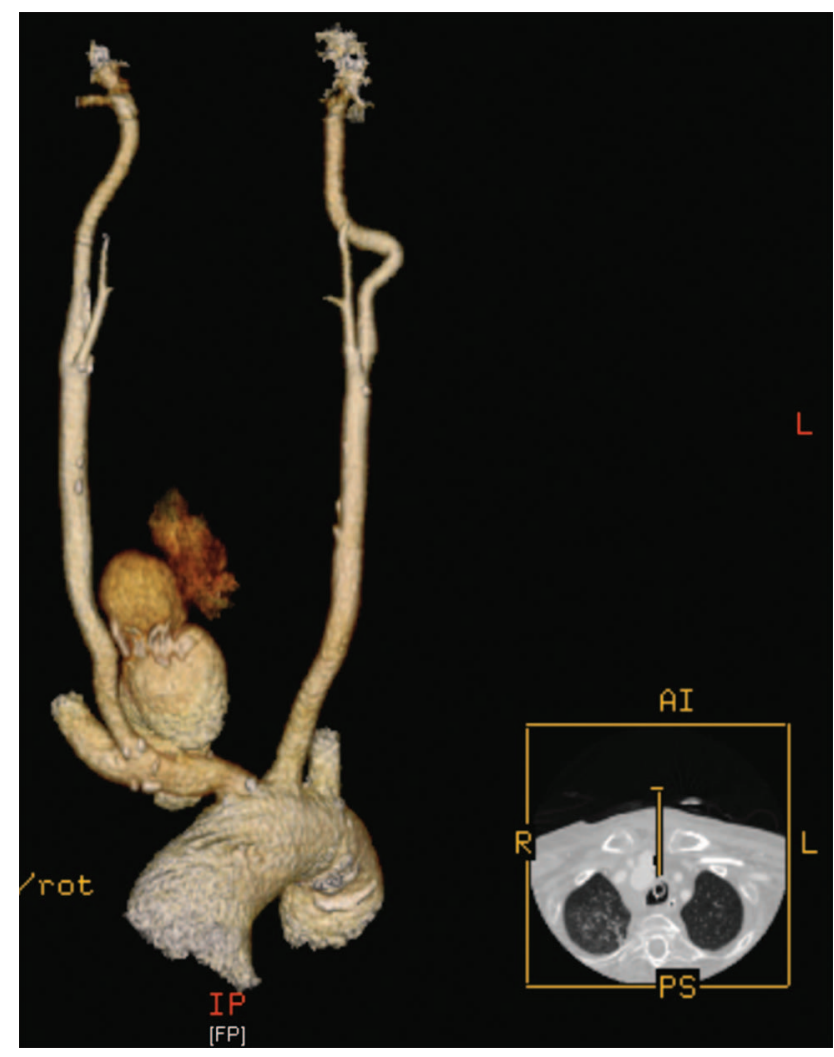

Fig. 2. Three-dimensional computed tomogram reconstruction of the great vessels extending into the neck, documenting a pseudoaneurysm of the right innominate artery. The pseudo-aneurysm measured $7.9 \mathrm{~cm}$ superior to inferior and $3.9 \mathrm{~cm}$ anterior to posterior.

catastrophic hemorrhage are usually delayed and are due to tracheo-innominate artery fistulas. Tracheo-innominate artery fistulas are an uncommon but life-threatening complication of tracheostomy placement, with mortality rates approaching $100 \% .^{3}$ The incidence of tracheo-innominate artery fistulas following PDT has been estimated to be $0.3 \% .{ }^{4}$ Interestingly, a fatal aortic arch laceration as an early complication of PDT has been reported. ${ }^{5}$ The postmortem examination of this patient determined that a $5-\mathrm{mm}$ tear in the upper part of the arch of the aorta was a direct result of damage incurred by PDT.

A PubMed search and an internet-based search engine (Google) failed to identify a prior description of a carotid artery laceration as a complication of PDT. Because deep venous access had not been attempted or placed on either side in our patient and because he had no evidence of bleeding in his neck prior to PDT, it seems likely that the carotid artery laceration was a complication of PDT. Exactly how the injury occurred to the carotid artery is not known. Because the patient was thin, the landmarks in the patient's neck were not obscured by fatty tissue. It could be that a dilator caused the injury either by being malpositioned or by piercing the trachea and the common carotid 
artery. Conversely, the patient's thinness may have placed him at an increased risk of injury to his great vessel because his carotid artery was in closer proximity to the operative field than usual. Because his airway was not examined via endoscope after the carotid artery bleeding occurred, the latter cannot be completely excluded.

Iatrogenic pseudo-aneurysm of the innominate artery has been described after subclavian vein deep venous access placement and as a complication of Hickman subcutaneous tunneled venous port insertion. ${ }^{6,7}$ However, a PubMed search and an internet-based search engine (Google) failed to identify a case of innominate artery pseudo-aneurysm as a complication of PDT even in large studies with multiple patients. As stated above, deep venous access had not been attempted or placed on either side in our patient. Therefore, it seems likely that his innominate artery pseudo-aneurysm was a complication of PDT. It may be that the same injury that resulted in the common carotid artery laceration caused the pseudo-aneurysm to form.

Early identification and appropriate surgical intervention are required when these complications occur. Our patient underwent emergent exploration of his neck with repair of his common carotid artery laceration. The was no physical finding to suggest an innominate artery pseudoaneurysm, such as a pulsatile mass, palpable thrill, or superior vena cava syndrome. ${ }^{2}$ This complication was discovered incidentally by computed tomography angiography. The pseudo-aneurysm was subsequently repaired.

A recent multi-institutional analysis of tracheostomy complications demonstrated a significantly higher rate of early postoperative bleeding with PDT (6.6\%) compared to conventional tracheostomy (1.9\%). ${ }^{1}$ The authors determined that the use of outer flange tracheostomy tube sutures reduces this complication. It has also been suggested that hemorrhage due to variant vascular anatomy, such as an inferior thyroid vein, a high brachiocephalic vein, and an aberrant anterior jugular communicating vein, may be minimized by preprocedural ultrasound of the operative site. ${ }^{8}$ In some studies, operators had to change the access site of tracheal tube placement after preprocedural ultrasound to avoid injuries to vascular structures. ${ }^{8,9}$

In addition to avoiding injury to pretracheal vascular structures, ultrasound-guided PDT offers other advantages and helps to guide safe tracheostomy placement by allowing better understanding of the anatomy of neck. ${ }^{10}$ Ultrasound can identify the tracheal midline, the levels of the tracheal cartilaginous rings, and overlying or vulnerable adjacent structures, such as the thyroid gland and isthmus. ${ }^{11}$ Ultrasound allows direct observation of the tracheostomy tube placement into the trachea and may prevent cranial misplacement of the tracheostomy tube. ${ }^{12,13}$ Realtime ultrasound-guided PDT is reported to be easy to perform and appears to be accurate and safe, including in patients with morbid obesity and cervical spine precau- tions. ${ }^{9}$ Even so, real-time ultrasound-guided PDT is not routinely performed in the majority of hospitals. Unfortunately, there are currently no randomized controlled trials to establish the safety and efficacy of preprocedural or real-time intra-operative ultrasound-guided PDT compared to the current standard of care. ${ }^{13}$

\section{Conclusions}

PDT has become a common procedure and is practiced by a variety of specialists. Although early complications are typically not serious, life-threatening adverse events can occur. We hope that our report of a carotid artery laceration and innominate artery pseudo-aneurysm formation as a consequence of PDT will alert practitioners to the potential for undesirable incidents. Furthermore, we hope that our report will inspire clinicians to search for ways to minimize complications from this relatively noninvasive procedure.

\section{REFERENCES}

1. Halum SL, Ting JY, Plowman EK, Belafsky PC, Harbarger CF, Postma GN, et al. A multi-institutional analysis of tracheotomy complications. Laryngoscope 2012;122(1):38-45.

2. Shlugman D, Satya-Krishna R, Loh L. Acute fatal hemorrhage during percutaneous dilatational tracheostomy. Br J Anaesth 2003;90(4): 517-520.

3. Richter T, Gottschlich B, Sutarski S, Müller R, Ragaller M. Late life-threatening hemorrhage after percutaneous tracheostomy. Int $\mathrm{J}$ Otolaryngol 2011;890380.

4. El Solh, AA. Airway management in the obese patient. Clin Chest Med 2009;30(5):555-568.

5. Ayoub OM, Griffiths MV. Aortic arch laceration: a lethal complication after percutaneous tracheostomy. Laryngoscope 2007;117(1): 176-178.

6. Philip RR, Boston US, Ballweg JA, Goldberg SP, Knott-Craig CJ. Iatrogenic pseudoaneurysm of the innominate artery in a neonate. J Card Surg 2012;27(2):242-244.

7. Petrocheilou G, Kokkinis C, Stathopoulou S, Fragopoulou L, Mihos P, Papadaki PJ, Vlychou M. Iatrogenic pseudoaneurysm of the brachiocephalic artery: a rare complication of Hickman line insertion. Int Urol Nephrol 2008;40(4):1107-1110.

8. Muhammad JK, Major E, Wood A, Patton DW. Percutaneous dilatational tracheostomy: haemorrhagic complications and the vascular anatomy of the anterior neck. A review based on 497 cases. Int J Oral Maxillofac Surg 2000;29(3):217-222.

9. Rajajee V, Fletcher JJ, Rochlen LR, Jacobs TL. Real-time ultrasound-guided percutaneous dilatational tracheostomy: a feasibility study. Crit Care 2011;15(1):R67.

10. Guinot PG, Zogheib E, Petiot S, Marienne JP, Guerin AM, Monet P, et al. Ultrasound-guided percutaneous tracheostomy in critically ill obese patients. Crit Care 2012;16(2):R40.

11. Gilbey P. Fatal complications of percutaneous dilatation tracheostomy. Am J Otolaryngol 2012;33(6):770-773.

12. Kristensen MS. Ultrasound in the management of the airway. Acta Anaesthesiol Scand 2011;55(10):1155-1173.

13. Rudas M, Seppelt I. Safety and efficacy of ultrasound before and during percutaneous dilatation tracheostomy in adult patients: a systemic review. Crit Care Resusc 2012;14(4):297-301. 